\title{
Magneto-exciton in single and coupled type II quantum dots
}

\author{
K. L. Janssens \\ Departement Natuurkunde, Universiteit Antwerpen (UIA), Universiteitsplein1, \\ B-2610 Antwerpen, Belgium
}

(November 13, 2018)

We studied the exciton energy in a type II quantum disk as a function of the magnetic field, disk radius $R$ and height $d$. We found angular momentum transitions for dots with $d>>$ $2 R$. Application of an electric field perpendicular to the disk showed a non-linear Stark shift. In the case of three vertically coupled dots angular momentum transitions were found for small interdot distances which disappeared with increasing interdot distance.

PACS: 73.21.La, 71.35.Ji, 85.35.Be

\section{INTRODUCTION}

Self-assembled quantum dots, as realized by the Stranski-Krastanow growth mode, have attracted considerable interest during the last decade. Most of this interest was dedicated to the study of type I quantum dots 近田, e.g. InAs/GaAs, where both electron and hole are confined in the dot. Also very interesting, though less studied, are the type II quantum dots [5 8], where the quantum dot forms an antidot for one of the carriers, e.g. for the holes in typically the InP/GaInP system or the electrons in e.g. GaSb/GaAs.

In the first part of this work, we focus our attention on the properties of an exciton in a single InP/GaInP quantum dot, where the hole is located in the barrier material. Our quantum dot is modeled as a quantum disk, with radius $R$ and thickness $d$ and strain effects are neglected. As a confinement potential, we take a hard wall of finite height. Furthermore, we apply an external magnetic field in the growth direction. To solve the problem, we use a Hartree-Fock (HF) mesh calculation, which allows very flexible solutions.

The second part of this work was dedicated to the study of an exciton in three vertically coupled identical dots with an interdot distance $d_{d}$.

\section{THEORETICAL MODEL}

We obtained the exciton energy and wavefunction by solving the HF single particle equations within the effective mass approximation (with $m_{e}$ and $m_{h}$ the effective electron and hole masses, respectively, $r_{e, h}=$ $\sqrt{x_{e, h}^{2}+y_{e, h}^{2}}, \omega_{c, e}=e B / m_{e}$ and $\left.\omega_{c, h}=e B / m_{h}\right)$. The $\mathrm{HF}$ single particle equations can be written as

$$
\begin{array}{r}
{\left[-\frac{\hbar^{2}}{2 m_{e}} \frac{1}{r_{e}} \frac{\partial}{\partial r_{e}}\left(r_{e} \frac{\partial}{\partial r_{e}}\right)-\frac{\hbar^{2}}{2 m_{e}} \frac{\partial}{\partial z_{e}}+\frac{\hbar^{2}}{2 m_{e}} \frac{l_{e}^{2}}{r_{e}^{2}}+\frac{l_{e}}{2} \hbar \omega_{c, e}+\frac{1}{8} m_{e} \omega_{c}^{2}\right.} \\
\left.+V_{e}\left(r_{e}, z_{e}\right)-\frac{e^{2}}{4 \pi \epsilon} \int \frac{\rho_{h}\left(r^{\prime}, z^{\prime}\right)}{\left|\mathbf{r}-\mathbf{r}^{\prime}\right|} d \mathbf{r}^{\prime}\right] \psi_{e}\left(r_{e}, z_{e}\right)=\epsilon_{e} \psi_{e}\left(r_{e}\right. \\
{\left[-\frac{\hbar^{2}}{2 m_{h}} \frac{1}{r_{h}} \frac{\partial}{\partial r_{h}}\left(r_{h} \frac{\partial}{\partial r_{h}}\right)-\frac{\hbar^{2}}{2 m_{h}} \frac{\partial}{\partial z_{h}}+\frac{\hbar^{2}}{2 m_{h}} \frac{l_{h}^{2}}{r_{h}^{2}}-\frac{l_{h}}{2} \hbar \omega_{c, h}+\frac{1}{8} m_{h} \omega_{c}^{2}\right.} \\
\left.+V_{h}\left(r_{h}, z_{h}\right)-\frac{e^{2}}{4 \pi \epsilon} \int \frac{\rho_{e}\left(r^{\prime}, z^{\prime}\right)}{\left|\mathbf{r}-\mathbf{r}^{\prime}\right|} d \mathbf{r}^{\prime}\right] \psi_{h}\left(r_{h}, z_{h}\right)=\epsilon_{h} \psi_{h}\left(r_{h}\right.
\end{array}
$$

with $\rho_{e}\left(r^{\prime}, z^{\prime}\right)$ and $\rho_{h}\left(r^{\prime}, z^{\prime}\right)$ respectively the electron and hole densities. As confinement potentials, we take hard walls of finite height, i.e.

$V_{e(h)}\left(r_{e(h)}, z_{e(h)}\right)=\left\{\begin{array}{c}V_{e(h)}, \quad r_{e(h)}>R \text { and }\left|z_{e, h}\right|>d / 2, \\ 0, \text { otherwise, }\end{array}\right.$

with $R$ the radius of the disk and $d$ its thickness.

The equations have to be solved self-consistently, which is done iteratively. We start with the free electron solution because in the absence of any Coulomb interaction only the free electron is confined. The Hartree integrals are integrated numerically

$$
\int \frac{\rho\left(r^{\prime}, z^{\prime}\right)}{\left|\mathbf{r}-\mathbf{r}^{\prime}\right|} d \mathbf{r}^{\prime}=4 \int d z^{\prime} \int \frac{\rho\left(r^{\prime}, z^{\prime}\right) r^{\prime}}{\sqrt{\left(r+r^{\prime}\right)^{2}+\left(z-z^{\prime}\right)^{2}}} \mathcal{K}\left(\frac{4 r r^{\prime}}{\left(r+r^{\prime}\right)^{2}+(z}\right.
$$

where $\mathcal{K}(x)$ is the complete elliptic integral of the first kind. More details about the calculation and numerical implementation of this integral is given in [7].

After convergence of the iteration procedure, the total energy is obtained as follows

$$
E_{\text {exciton }}=\epsilon_{e}+\epsilon_{h}+\frac{e^{2}}{4 \pi \epsilon} \iint \frac{\rho_{e}(r, z) \rho_{h}\left(r^{\prime}, z^{\prime}\right)}{\left|\mathbf{r}-\mathbf{r}^{\prime}\right|} d \mathbf{r} d \mathbf{r}^{\prime},
$$

where $\epsilon_{e(h)}$ is the electron (hole) single particle energy and $\rho_{e(h)}$ is the electron (hole) density.

\section{RESULTS}

First we considered the exciton in a single quantum disk. We took the following material parameters: $m_{e}=$ 
$0.077 m_{0}, m_{h}=0.60 m_{0}, V_{e}=250 \mathrm{meV}, V_{h}=-50 \mathrm{meV}$ and $\epsilon=12.61$. The influence of the disk parameters (i.e. radius and height) were studied in the absence of a magnetic field which resulted in the phase diagram shown in Fig. 1. The solid curve corresponds to the probability to find $50 \%$ of the hole wavefunction at the radial boundary of the disk. Here the probability was calculated as

$$
P_{\text {side }}=2 \pi \int_{-\infty}^{\infty} d z_{h} \int_{R}^{\infty} r_{h}\left|\Psi_{h}\left(r_{h}, z_{h}\right)\right|^{2} d r_{h} .
$$

One can distinguish between two regimes: a) the disklike regime $(d<<2 R)$ where less than $50 \%$ of the hole wavefunction is situated at the radial boundary, and b) the pillar-like regime $(d>>2 R)$ for the case of $P_{\text {side }}>$ $50 \%$. In order to give a more visual picture, we made contourplots of the hole wavefunction for the two regimes, shown as insets to Fig. 1 for the cases of $R=4 \mathrm{~nm}$, $d=12 \mathrm{~nm}$ and $R=12 \mathrm{~nm}, d=4 \mathrm{~nm}$. The two regimes are a consequence of the fact that the hole tends to sit as closely to the electron as possible. Therefore, when $d<<2 R$, the hole will prefer to sit above and below the dot, whereas for $d>>2 R$ the hole will be located at the radial boundary. Because the volume available for the hole above and below the dot is smaller than at the radial boundary we found that the $50 \%$ division line is not given by $d \simeq 2 R$ but could be approximated by $d \simeq-8+2 R$ in the investigated $(d, R)$ range as presented in Fig. 1.

An exciton will behave differently when a magnetic field is applied along the $z$-direction depending on the position of the quantum dot in the phase diagram. Fig. 2 shows the exciton energy as function of the magnetic field for a disk-like system (Fig. 2(a), $R=12 n m, d=4 n m$ ) and for a pillar-like system (Fig. 2(b), $R=4 n m, d=$ $12 \mathrm{~nm})$. We find that in the first case the angular momentum of the hole, $l_{h}$, remains zero over the whole $B$-regime, whereas for the latter case we find angular momentum transitions with increasing magnetic field. These follow from the fact that, when the hole is sitting at the radial boundary, the magnetic field pushes the hole closer to the disk boundary, making it energetically more favourable to jump to a higher $l_{h}$ state.

In a next step, we consider the influence of an electric field, applied along the $z$-direction. Application of such an electric field leads to a polarisation of the system. Following parameters were used: $R=10 \mathrm{~nm}, d=2 \mathrm{~nm}$, $V_{e}=250 \mathrm{meV}$ and $V_{h}=-50 \mathrm{meV}$. Fig. 3 depicts the dependence of the exciton energy on the applied electric field. We observe a shift towards lower energies with increasing $F$, which is the so called Stark shift. The inset of Fig. 3 shows the behaviour of the hole wavefunctionwhich moves towards the bottom of the disk with increasing electric field. The Stark shift is almost entirely due to the shift of the hole. Note that the calculation is limited to very small values of the electric field. Since the hole is only confined to the dot by the Coulomb attraction to the electron, the system becomes fastly unbound at higher fields.
Finally, the system of three vertically coupled dots was studied under the influence of a magnetic field. This system is especially interesting as it allows to construct a pillar-like structure. We now have an extra parameter to vary, namely the interdot distance $d_{d}$. When $d_{d}$ is very small, there will be not enough space for the holes to sit between the quantum dots. Therefore, when the total stack height (i.e. three times the dot thickness plus two times the interdot distance) is larger than the disk diameter, the hole will prefer to sit at the radial boundary, exactly as in the case of the pillar-like system for a single dot. For our numerical work we used the parameters: $R=8 \mathrm{~nm}, d=3 \mathrm{~nm}, V_{e}=250 \mathrm{meV}$ and $V_{h}=-30 \mathrm{meV}$. Fig. 4 shows the exciton energy for $d_{d}=3 \mathrm{~nm}$ as function of the magnetic field, and we see again the appearance of angular momentum transitions. The insets of Fig. 4 show contourplots for the hole wavefunction at respectively $B=0 T$, where $l_{h}=0$, and $B=50 T$, where $l_{h}=4$ is the groundstate. At $B=0 T$, part of the hole wavefunction is still between the dots. By applying a strong magnetic field, a large part of the hole wavefunction is pushed between the dots. However, there is not enough space, and therefore it is more favourable to jump to a higher $l_{h}$ state. When we increase now the interdot distance $d_{d}$ to $5.5 \mathrm{~nm}$, we give the hole more space between the dots and in this way create a transition to a regime without angular momentum transitions (like the disk-like regime). This is depicted in Fig. 5, where the insets show the contourplots of the hole wavefunction at respectively $B=0 T$ and $B=50 T$. We find no angular momentum transition, and we see that at $B=50 T$ the hole is entirely situated between the dots.

\section{CONCLUSIONS}

We studied the exciton energy in a single type II quantum disk as a function of the magnetic field. We made a distinction between a disk-like regime, where the hole angular momentum remains zero, and a pillar-like regime, where angular momentum transitions appear with increasing magnetic field. Applying an electric field results in a Stark shift towards lower energies and a strong polarisation of the hole wavefunction. Finally we studied the case of three vertically coupled dots. For very small interdot distances, this systems resembles the pillar-like regime, with the occurrence of angular momentum transitions. When increasing the interdot distance, we found no longer $l_{h}$ transitions, because of the possibility for the hole to sit between the dots.

\section{ACKNOWLEDGEMENTS}

This work is supported by the Flemish Science Foundation (FWO-Vl), The Inter-University Attraction Poles research program (IUAP-IV) and the University of 
Antwerp (GOA and VIS). K. L. J. is supported by the "Instituut voor de aanmoediging van Innovatie door Wetenschap en Technologie in Vlaanderen" (IWT-Vl) and B. P. is a post-doctoral researcher with the FWOVl. Discussions with M. Tadic, M. Hayne and V. Moshchalkov are gratefully acknowledged.

* corresponding author, electronic mail: karenj@uia.ua.ac.be

- Electronic mail: bpartoen@uia.ua.ac.be

$\dagger \quad$ Electronic mail: peeters@uia.ua.ac.be

[1] P.D. Wang, J.L. Merz, S. Fafard, R. Leon, D. Leonard, G. Medeiros-Ribeiro, M. Oestreich, P.M. Petroff, K. Uchida, N. Miura, H. Akiyama, and H. Sakaki, Phys. Rev. B 53, 16458 (1996).

[2] M. Bayer, A. Schmidt, A Forchel, F. Faller, T.L. Reinecke, P.A. Knipp, A.A. Dremin, and V.D. Kulakovskii, Phys. Rev. Lett. 74, 3439 (1995).

[3] O. Stier, M. Grundmann, and D. Bimberg, Phys. Rev. B 59, 5688 (1999).

[4] K.L. Janssens, F.M. Peeters, and V.A. Schweigert, Phys. Rev. B 63, 205311 (2001).

[5] M. Hayne, R. Provoost, M.K. Zundel, Y.M. Manz, K. Eberl, and V.V. Moshchalkov, Phys. Rev. B 62, 10324 (2000).

[6] M. Sugisaki, H.-W. Ren, K. Nishi, S. Sugou, T. Okuno, and Y. Masumoto, Physica B 256-258, 169 (1998).

[7] K.L. Janssens, B. Partoens, and F.M. Peeters, accepted for publication in Phys. Rev. B.

[8] Ph. Lelong, K. Suzuki, G. Bastard, H. Sakaki, and Y. Arakawa, Physica E 7, 393 (2000).

FIG. 1. Phase diagram of the probability for the hole wavefunction to sit at the radial border of the disk, as a function of both $R$ and $d$. The insets show the contourplots of the hole wavefunction, at respectively $R=4 \mathrm{~nm}, d=12 \mathrm{~nm}$ and $R=12 \mathrm{~nm}, d=4 \mathrm{~nm}$. The dashed lines indicate the boundary of the disk.

FIG. 2. The exciton energy as a function of the magnetic field, for (a) a disk-like structure and (b) a pillar-like structure.

FIG. 3. The exciton energy as a function of a vertical electric field. The inset shows the hole wavefunction for a field of $F=1 \mathrm{kV} / \mathrm{cm}$.
FIG. 4. The exciton energy as a function of the magnetic field for three coupled dots, with $d_{d}=3 \mathrm{~nm}$. The insets are contourplots of the hole wavefunction at respectively $B=0 T$ and $B=50 T$.

FIG. 5. The same as Fig. 4, but now for $d_{d}=5.5 \mathrm{~nm}$. 


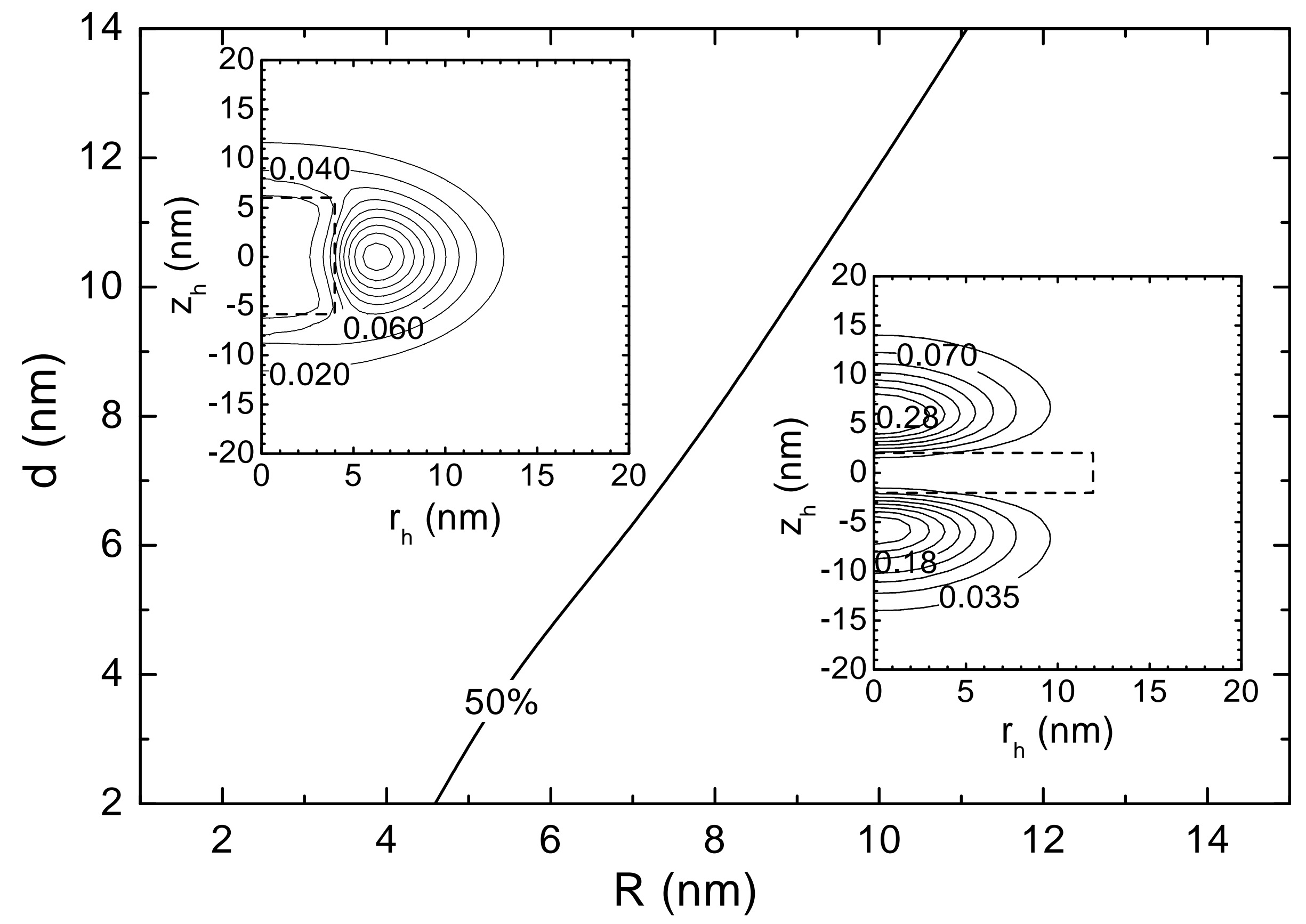




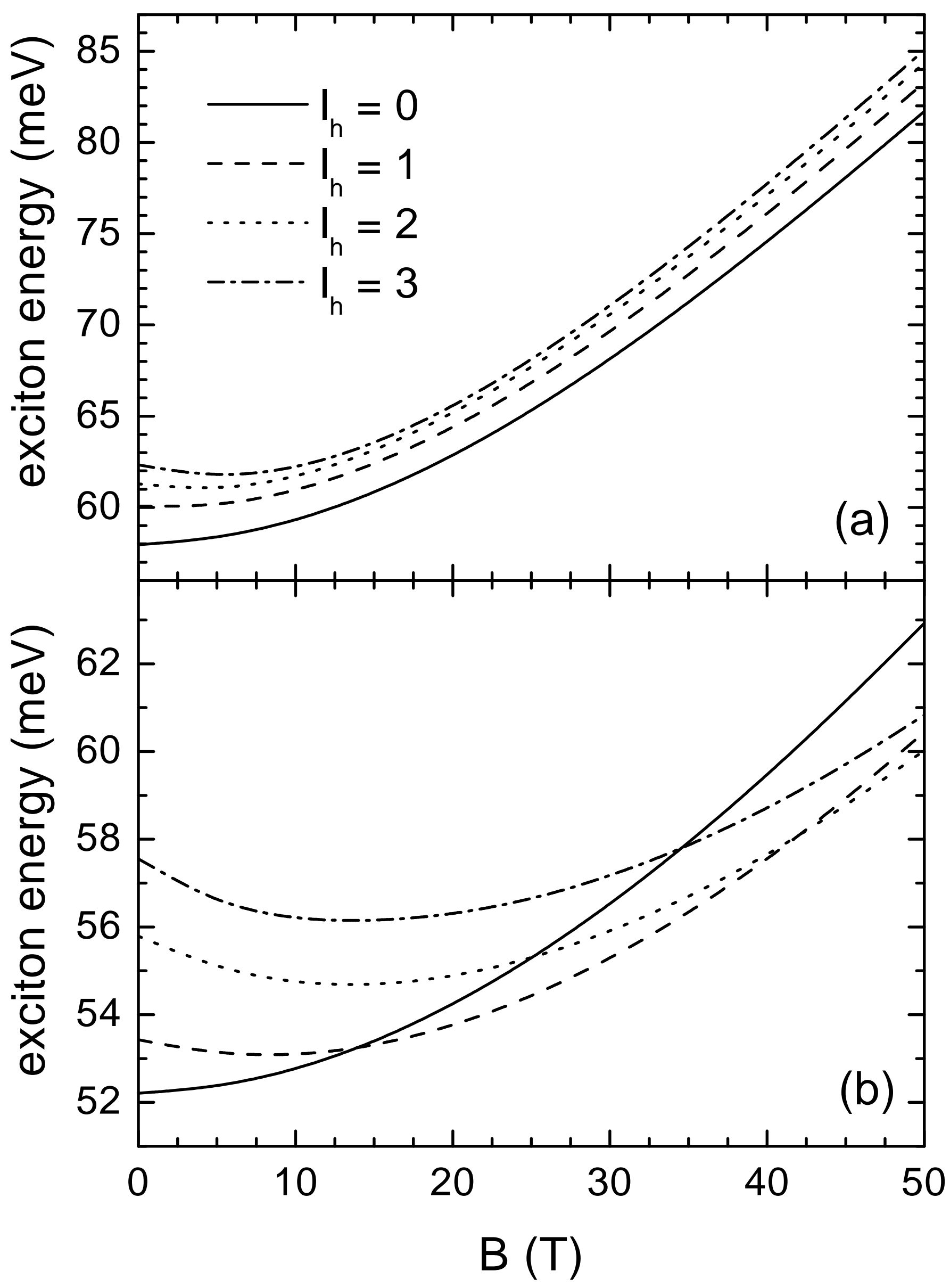




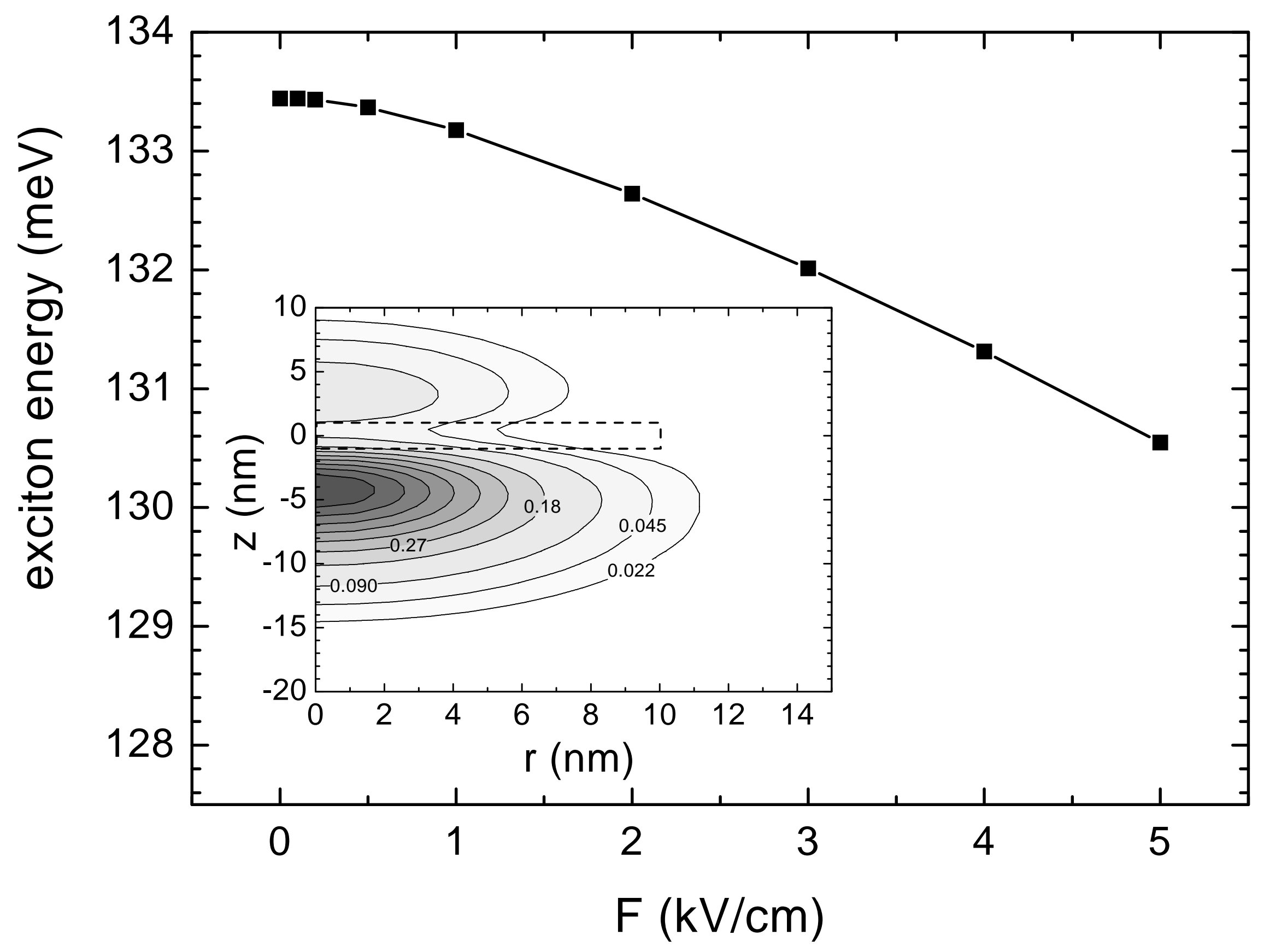




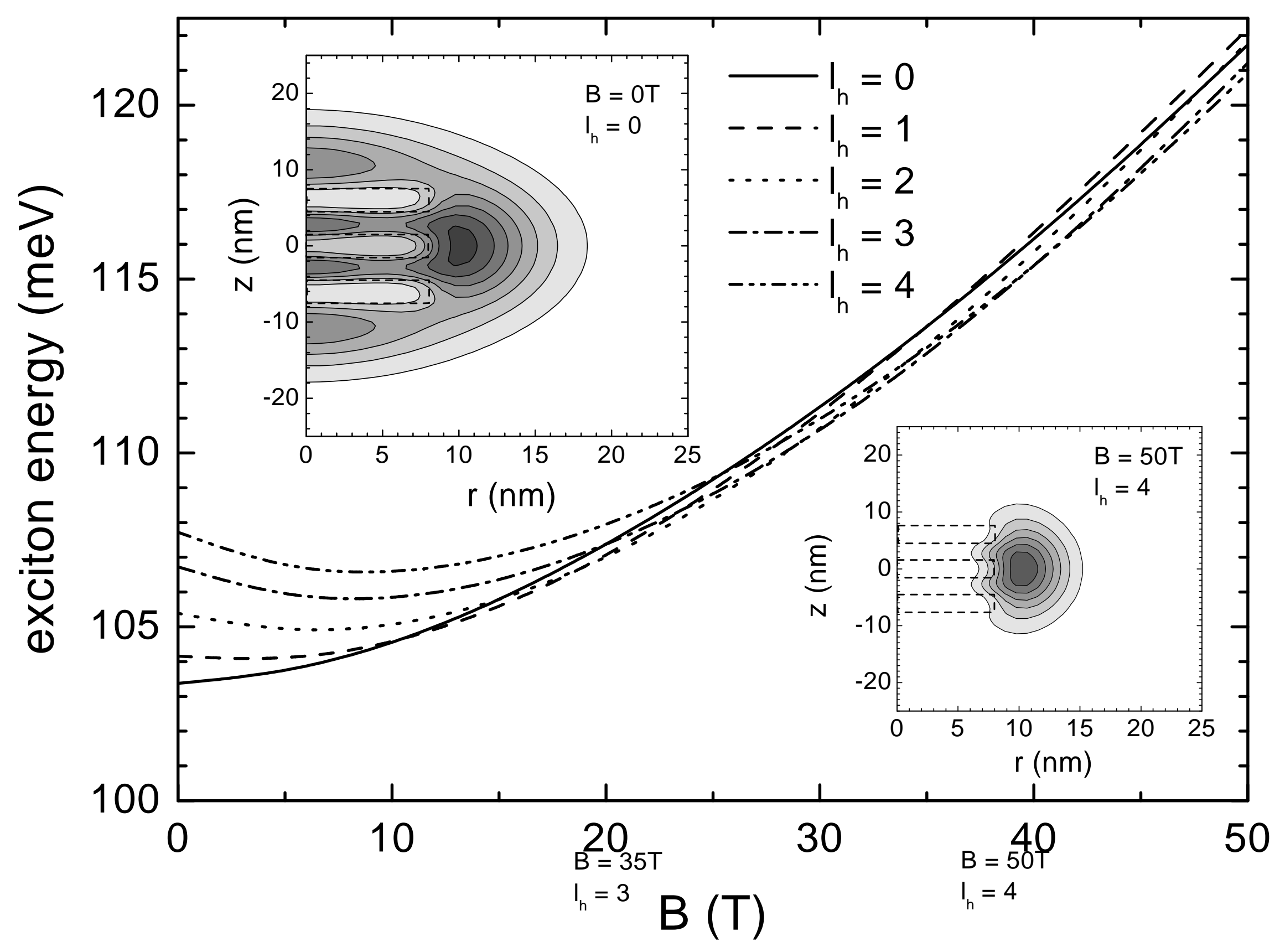




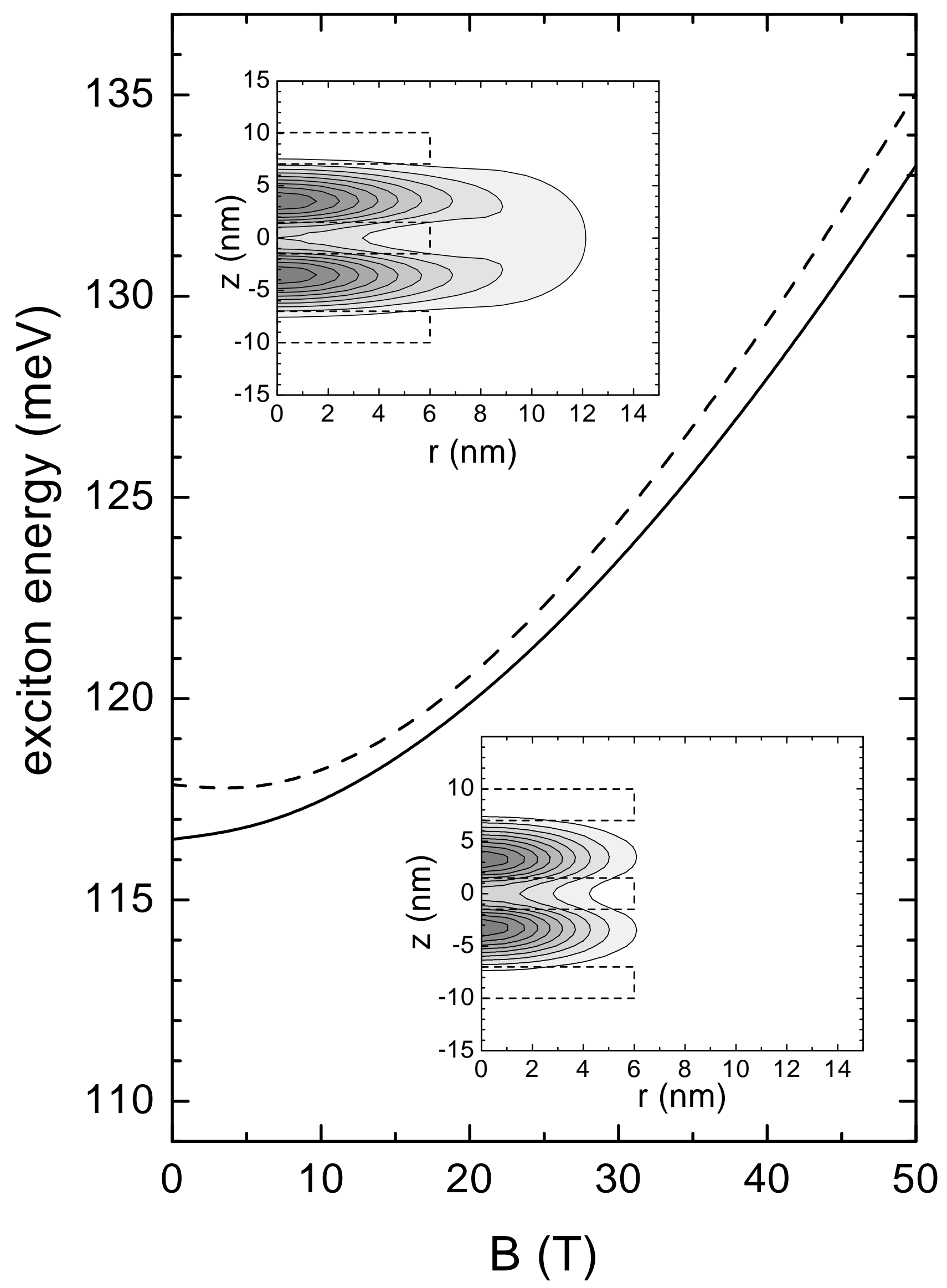

